\title{
Trends and Technologies used for Mitigating Energy Efficiency Issues in Wireless Sensor Network
}

\author{
Jyothi A.P \\ Research Scholar \\ Visvesvaraya Technological University \\ Belgaum, India
}

\author{
Usha Sakthivel, Ph.D \\ Prof. \& HOD: Dept. of CSE, \\ Rajarajeswari College of Engg. \\ Bangalore, India
}

\begin{abstract}
In the recent times, various applications are conceptualized where wireless sensor network (WSN) is used either as a subnetwork or as a complete domain. WSN having its unique characteristics, because of that the applicable protocols for congestion control, routing and security require distinguished mechanism as compared to other wireless networks such as WLAN, MANET, etc. One of the most irreversible resources is battery power. Since year 2000 a project called $\mu$ AMS in Massachusetts Institute of technology (MIT), where Wendi Heizelman has introduced a communication protocol called Low Energy Adaptive clustered Hierarchy (LEACH). Since then, till today enormous amount of research schemes have been suggested to have different layers protocols in WSN, with optimal use of energy. This paper aims to study, investigate and analyze various contributions, limitations, technology used towards energy optimization based protocol development in WSN. The outcome of this paper will be quite valuable fro academicians, industries and researchers as a one hand tool to understand future research directions.
\end{abstract}

\section{Keywords}

Battery, Energy Efficiency, LEACH, Network Lifetime, Wireless Sensor Network

\section{INTRODUCTION}

The area of wireless sensor network is one the constant attention of the research community owing to its potential advantages and the persistent issues associated with it [1]. Basically, wireless sensor network consist of number of sensor nodes that are distributed around the region which is required to be monitored or certain data are required to be captured. A sensor node is a small device that has the capability of sensing data pertaining to physical attributes like heat, moisture, motion, pressure, smoke etc. Theoretical study suggests that there are three types of nodes considered in research work e.g. cluster head, member node, and sink node. The member node is responsible for capturing the raw data, which by using TDMA scheduling forwards the data to the cluster head $(\mathrm{CH})$. The prime responsibility of $\mathrm{CH}$ is to perform data aggregation and thereby forward the aggregated and non-redundant data either to the sink directly (single hop) or it is neighbor $\mathrm{CH}$ (multi-hop). Both from theoretical as well as practical study viewpoint, wireless sensor network is categorized into two types e.g. homogenous (application with similar types of sensor node configuration) and heterogeneous wireless sensor network (application with different types of sensor node configuration). The applications of wireless sensor network are found in industrial monitoring, health monitoring, habitat monitoring etc. While analyzing the performance of the wireless sensor network, it is equally important to consider the actual hardware and radio charecteristics of a sensor node. Essentially, sensor node has a very limited computational capability with availability of less resource. One of another critical resource constraint is battery life. Studied carried out in last decade have essentially understood that energy is one of the responsible factor that are tough to be controlled or optimized. The negative impact of energy can affect routing behaviour, quality of service behaviour, as well as security. Network performance highly degrades when mobility is considered. Energy is one prime requirements of data aggregation techniques as majority of the research techniques considers selection of $\mathrm{CH}$ based on residua energy. Also $\mathrm{CH}$ consumes large amount of energy when it aggregates and forward the massive data to the sink. A sensor node has a typical charecteristics when it comes to energy drainage. Energy is depleted not only in the active stage but also in passive stage (state transition from sleep to active in sleep scheduling algorithm). A significant study performed by Heizelman [2] by introduced an energy efficient routing protocol called as LEACH that has considered the design principle of radio-energy model based on standard antenna theory, which is still widely applicable in various hardware designs. Therefore, LEACH is one of the most widely used standards of energy efficient routing protocol till date. However, LEACH is also accompanied by various drawbacks.

Hence, various studies have been proposed in past that has introduced a technique to mitigate the energy efficient, where majority of the techniques circles around routing protocols itself. It should be known that for various applications that works on remote areas requires the node to be operated for extra long time as it is not possible for replacing the battery very often. Moreover, different components of hardware have different energy requirement for performing data aggregation task. Hence, this paper discusses some of the significant studies carried out in past that have introduced various techniques of energy efficiencies in wireless sensor network. The paper deeply reviews the techniques presented in past decades as well as some recent years and extracts the research gaps based on evaluation Section II discusses about the challenges of the wireless sensor network followed by Section III that discusses about existing research trends. Section IV discusses about the research gap, while Section V discusses about the summary of the paper.

\section{CHALLENGES}

The prominent challenges encountered at the time of data aggregation process in WSN are as follows [3]:

\subsection{Energy Consumption}

As energy consumption determines the lifetime of the Wireless sensor network. Hence it becomes the major issue in Wireless sensor network. Most of sensor nodes use battery power as their energy source. The sensor network can be deployed in hazards conditions so it becomes difficult to change their batteries or provider the energy so there is requirement of developing the networks which efficiently use the battery as energy. The energy consumption depends upon major operations of the sensor nodes which are Sensing, Communication, Data processing. The large amount of energy is consumed during the communication. Batteries with high power as rechargeable batteries like solar panel can be used in some wireless sensor networks. 


\subsection{Data Gathering}

It is a major issue in Wireless sensor network as it determines the lifetime of network. Data gathering is task of collecting the data from different sensors by removing the redundant data. The information collected must be delivered to sink node without loss of information. Sensors node forward their own packet and also forward packets produced by others sensors, hence tends to consume more energy and form the energy holes near the sinks.

\subsection{Query Processing}

Query processing involves the answering the query from sink by gathering the information from other sensor nodes. The storage node stores the data from other nodes and answers the query from sink in this way it is a two tier architecture and storage node act as intermediate node.

\subsection{Scheduling}

It is also a important factor as it determines that for which time period the sensor node will in which node either sleep, active or on standby mode. Scheduling also plays important role for coverage and connectively. By using proper scheduling we can reduce the energy consumption.

\subsection{Scalability}

There are number of nodes in wireless sensor network. Depending on the requirements of the application number of nodes can be increased or decreased. Wireless sensor network should be such that it should be able to accept new node and coordinate them with existing nodes.

\subsection{Reliability}

A node may fail due to various reasons. The failure of one node should not affect the overall performance of the Wireless sensor network. Information delivery application information deliveries dependent have issues: 1) Packet reliability: applications that are less sensitive require successful transmission of all packets. 2) Event reliability: applications require only successful event detection and not successful transmission of all packets. 3) Destination related reliability: applications have to send message to node that lies is specific area of network.

Table 1 exhibits the inferences of most widely used energy efficient routing protocols found in literature.

Table 1: Summary of Existing Routing Protocols used for energy efficiency in WSN [3]

\begin{tabular}{|l|l|}
\hline Protocols & \multicolumn{1}{c|}{ Charecteristics } \\
\hline LEACH & $\begin{array}{l}\text { Pros: i) Unique selection of node as CH, ii) Using } \\
\text { TDMA scheduling allows less extent of packet } \\
\text { collision, iii) Higher extent of energy preservation. }\end{array}$ \\
\cline { 2 - 3 } & $\begin{array}{l}\text { Cons: i) Not suitable for large networks, ii) Extra } \\
\text { overhead }\end{array}$ \\
\hline HEED & $\begin{array}{l}\text { Pros: i) Distributed clustering method, ii) Provides } \\
\text { better load balancing, iii) Supports multi-hop } \\
\text { communication between Sink and CHs }\end{array}$ \\
\cline { 2 - 2 } & $\begin{array}{l}\text { Cons: i) Consequent overhead during clustering, ii) } \\
\text { Early death of CH who are in close proximity of } \\
\text { sink, iii) not much control on energy dissipation }\end{array}$ \\
\hline DWEHC & $\begin{array}{l}\text { Pros: i) complete distributive clustering technique, } \\
\text { ii) Less energy consumption in intra-cluster and } \\
\text { inter-cluster routing because of stable CHs } \\
\text { distribution, iii) Faster processing of clustering } \\
\text { mechanism. }\end{array}$ \\
\hline
\end{tabular}

\begin{tabular}{|l|l|}
\hline \multirow{2}{*}{} & $\begin{array}{l}\text { Cons: i) Supports on one-hop network, ii) doesn't } \\
\text { supports large scale network, ii) introduces larger } \\
\text { control overhead }\end{array}$ \\
\hline PEGASIS & $\begin{array}{l}\text { Pros: i) Better version of LEACH, ii) supportability } \\
\text { of different network sizes, iii) proper distribution of } \\
\text { energy among the nodes }\end{array}$ \\
\cline { 2 - 3 } & $\begin{array}{l}\text { Cons: i) Support only network with time- } \\
\text { fluctuating topology, ii) Consume energy, iii) Leads } \\
\text { to overheads and network blockage, iv) Doesn't } \\
\text { address scalability issues of WSN }\end{array}$ \\
\hline PANEL & $\begin{array}{l}\text { Pros: i) energy-efficient protocol, ii) Efficient load } \\
\text { balancing, iii) Supports both synchronous and } \\
\text { asynchronous applications }\end{array}$ \\
\cline { 2 - 2 } & $\begin{array}{l}\text { Cons: i) Doesn't support dynamic topology, ii) } \\
\text { dependability of geographical location information. }\end{array}$ \\
\hline
\end{tabular}

\section{EXISTING RESEARCH TRENDS}

This section discusses about the existing trends of the research work carried out in the direction of achieving energy efficiencies in wireless sensor network. After excavating various significant research papers, it was found that various techniques and methodologies were adopted to overcome the energy issues in wireless sensor network (Refer Table 2).

Most recently the adoption of opportunistic routing principles were witnessed in the study conducted by Mao et al. [4], who have introduced a strategy to minimize the energy depletion in wireless sensor network using cost based approach for adjusting the energy using 4 distinct algorithms. Evaluated on TinyOS using CSMA MAC protocol, the study has been evaluated using total transmissions, received packets, energy consumption, duplication of packet, packet loss ratio, and delay. The authors have performed benchmarking of their studies with ExOR [5], which is one of the prior existing opportunistic routing protocols. Adoption of MAC protocol was also observed in the work carried out by Halkes and Langendoen [6]. The protocol was designed based on node synchronization to solve the energy drainage in TinyOS. The outcome of the study was evaluated using delivery ratio, energy consumption and latency. The benchmarking was done by adopting T-MAC and LMAC protocols. The work done by Halkes and Langendoen [6] is done for dense network, however, there is also a significant study found for energy efficiency towards low-density network. One of such study was carried out by Francesco et al. [7], who have adopted a strategy by incorporating mobile data collector for data aggregation process in Java. Similar patterns of experiments by adopting MAC protocol was also seen in the study of Tang et al. [8], where the authors have attempted to forecast the receiver wake-up channel and time using TinyOS. The technique was evaluated using duty cycle, latency, and delivery ratio.

Aziz et al. [9] have presented their framework for energy efficiency in wireless sensor network considering the case study of real-time image transmission. Implemented over FPGA, the study was tested with amount of energy being drained during transmission process. Work in similar direction of energy efficiency along with source location privacy was witnessed in the study of Long et al. [10]. The study has adopted tree-based routing techniques to ensure energy efficiency using OMNet++. The outcomes have been evaluated using delay, network lifetime, number of established routes, and mean path length.

Literature have also witnessed some unique and potential studied considering the sophisticated radio design of sensor nodes for 
evaluating energy effectiveness. One of such study was carried out by Islam et al. [11], where the author have presented a mathematical model based on cross-layer approach using NS2. The energy efficiency was closely observed by analyzing throughout and energy consumption. Similarly, another unique work was found in the literature in the study carried out by Takaishi et al. [12], where the author have presented a novel mobile sink routing to minimize energy consumption in wireless sensor network in $\mathrm{C}++$. The outcome of the study was evaluated using energy consumed and efficiency. Another interesting study was seen in the work presented by Damaso et al. [13] on the basis of simulation using CPN tools [14]. Most recently, the network lifetime maximization was investigated by Ashwini and Raju [15], where the authors have identified multiple attributes responsible for energy depletion in wireless sensor network. The most recent version of the energy efficient routing protocol called as Q-LEACH was introduced by Manzoor et al. [16] in 2013. The outcomes were significant evaluated using number of alive nodes, number of dead nodes, data packets, and number of clusters. The benchmarking was also done with LEACH protocol to fine significantly better outcomes

Apart from the above discussed studies, there are also some set of studies that uses a specific technology to ensure energy effectiveness. Hence, some of the frequently adopted techniques found in the existing literatures are as follows (Refer Table 3):

\subsection{Neural Network}

Neural network has been consistently adopted by many researchers to perform the iterative computing along with training and validation to explore the best outcome of energy conservations schemes. Guan and $\mathrm{Lu}$ [17] have used multilayered backpropagation learning technique for minimizing energy consumption. Hosseingholizadeh [18] used neural network for energy efficient routing. Another significant study was conducted

Table 2: Summary of the Contribution of Recent Research Attempts in Energy Efficiency

\begin{tabular}{|c|c|c|c|}
\hline Author & Techniques Adopted & Performance parameters & Inference \\
\hline Mao et al. [4] & $\begin{array}{l}\text { Cost based energy } \\
\text { adjustment }\end{array}$ & $\begin{array}{l}\text { total transmissions, received } \\
\text { packets, energy consumption, } \\
\text { duplication of packet, packet loss } \\
\text { ratio, and delay. }\end{array}$ & $\begin{array}{l}\text { Pros: benchmarked outcomes } \\
\text { Cons: Didn't benchmarked with LEACH, less } \\
\text { discussion on scalability issues }\end{array}$ \\
\hline $\begin{array}{l}\text { Halkes \& } \\
\text { Langendoen [6] }\end{array}$ & MAC-Protocol & $\begin{array}{lll}\text { delivery ratio, } & \text { energy } \\
\text { consumption and latency } & \end{array}$ & $\begin{array}{l}\text { Pros: support various network density } \\
\text { Cons: benchmarking was not done with energy } \\
\text { efficiency routing protocols }\end{array}$ \\
\hline $\begin{array}{l}\text { Francesco et al. } \\
\text { [7] }\end{array}$ & $\begin{array}{l}\text { Adaptive strategy of } \\
\text { incorporating mobile data } \\
\text { collector }\end{array}$ & Number of task execution, energy, & $\begin{array}{l}\text { Pros: ensure effective resource allocations, } \\
\text { adaptable to other operating condition and } \\
\text { mobility patterns } \\
\text { Cons: Not benchmarked with standard routing } \\
\text { protocols for energy efficiency }\end{array}$ \\
\hline Tang et al. [8] & $\begin{array}{l}\text { Multi-channel } \\
\text { Protocol }\end{array}$ & $\begin{array}{l}\text { Duty cycle, latency, and delivery } \\
\text { ratio. }\end{array}$ & $\begin{array}{l}\text { Pros: Supports multi-channel energy efficiency, } \\
\text { minimized duty cycle and delivery latency } \\
\text { observed. } \\
\text { Cons: Not benchmarked with standard routing } \\
\text { protocols for energy efficiency }\end{array}$ \\
\hline Aziz et al. [9] & $\begin{array}{l}\text { FPGA based object } \\
\text { extraction algorithm }\end{array}$ & Energy & $\begin{array}{l}\text { Pros: Tested in real-time, significant reduction } \\
\text { of energy } \\
\text { Cons: Outcome not benchmarked }\end{array}$ \\
\hline Long et al. [10] & Tree based routing & $\begin{array}{l}\text { delay, network lifetime, number } \\
\text { of established routes, and mean } \\
\text { path length. }\end{array}$ & $\begin{array}{l}\text { Pros: achieves security and energy efficiency. } \\
\text { Cons: Outcome not benchmarked }\end{array}$ \\
\hline
\end{tabular}

by Abbasi and Kamal [19] who have adopted neural network for predicting energy resources. Bahanfar et al. [20] have also used neural network to perform power optimization.

\subsection{Fuzzy Logic}

Just like neural network, adoption of fuzzy logic is also quite abundant in the literatures. Owing to primary issues of vagueness in problems or uncertainty in data, fuzzy logic is primarily adopted to get the best possible outcome from either incomplete [21] have used fuzzy logic to minimize communication overhead, which in-turn can reduce energy consumption. Jiang et al. [22] have used fuzzy logic to design a function for forecasting the inequality of residual energy of neighbors. Similar pattern of studies were also witnessed in the literature of Hadjila et al. [23]. For enhancing the election process of cluster head, fuzzy logic is extensively adopted by Mostafa et al. [24]. Fuzzy Logic was also adopted for minimizing congestion as studied in the work of Sonmez et al. [25]. Behzadi and Azad [26] have adopted fuzzy logic to achieve fault-tolerant properties in wireless sensor network

\subsection{Genetic Algorithm}

Known for its evolutionary techniques, genetic algorithm (GA) is one of another frequently adopted technique to obtain the elite results from the set of population. Fard et al. [27] used GA for energy efficient QoS Routing. The recent version of LEACH was enhanced using GA by Liu [28]. Studies on energy efficiency using GA was also seen in the work of Heidari [29], Zahhad et al. [30], and Mehrjoo et al. [31] most recently. Hence, it can be seen that genetic algorithm was used in many cases for energy efficiency in wireless sensor network where motive was to enhance the cluster based energy efficiency in routing protocol. data or uncertain data in wireless sensor network. Zarafshan et al. 


\begin{tabular}{|l|l|l|l|}
\hline Islam et al. [11] & $\begin{array}{l}\text { Mathematical modelling } \\
\text { using cross-layer }\end{array}$ & Throughout, energy consumption & $\begin{array}{l}\text { Pros: Real-time implementation } \\
\text { supportability on backpack radios. } \\
\text { Cons: Outcome not benchmarked, Impact of } \\
\text { proposed technique not evaluated w.r.t QoS } \\
\text { parameters extensively. }\end{array}$ \\
\hline $\begin{array}{l}\text { Taikaishi et al. } \\
{[12]}\end{array}$ & $\begin{array}{l}\text { Enhanced clustering } \\
\text { method }\end{array}$ & energy consumed, efficiency & $\begin{array}{l}\text { Pros: Supports data aggregation of Big Data } \\
\text { Cons: Outcome not benchmarked, Impact of } \\
\text { proposed technique not evaluated w.r.t QoS } \\
\text { parameters extensively. }\end{array}$ \\
\hline $\begin{array}{l}\text { Damaso et al. } \\
{[13]}\end{array}$ & $\begin{array}{l}\text { Simulation using CPN } \\
\text { tools }\end{array}$ & $\begin{array}{l}\text { energy } \\
\text { Pros: Good Analytical formulation } \\
\text { Cons: Outcomes not benchmarked, no } \\
\text { validation or discussion of applicability of the } \\
\text { analytical model in large network }\end{array}$ \\
\hline $\begin{array}{l}\text { Ashwini \& Raju } \\
{[15]}\end{array}$ & $\begin{array}{l}\text { Time approximation } \\
\text { algorithm for data } \\
\text { aggregation }\end{array}$ & $\begin{array}{l}\text { Time } \\
\text { Pros: identify time factor for resource } \\
\text { allocation } \\
\text { Cons: Outcomes not benchmarked }\end{array}$ \\
\hline $\begin{array}{l}\text { Manzoor et al. } \\
{[16]}\end{array}$ & $\begin{array}{l}\text { Cluster based energy } \\
\text { efficient routing }\end{array}$ & $\begin{array}{l}\text { number of alive nodes, number of } \\
\text { dead nodes, data packets, and } \\
\text { number of clusters }\end{array}$ & $\begin{array}{l}\text { Pros: enhanced version of LEACH } \\
\text { Cons: No discussion on scalability issues }\end{array}$ \\
\hline
\end{tabular}

Table 3: Summary of the frequently used techniques in Energy Efficiency

\begin{tabular}{|c|c|c|c|c|}
\hline & Authors & Techniques & Performance Parameters & Inference \\
\hline \multirow{4}{*}{ 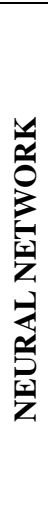 } & Guan \& Li [17] & $\begin{array}{l}\text { Multilayered } \\
\text { backpropagation }\end{array}$ & Energy cost, & $\begin{array}{l}\text { Pros: Enables intelligent decision } \\
\text { making for data quality assurance } \\
\text { Cons: Outcomes not benchmarked }\end{array}$ \\
\hline & $\begin{array}{l}\text { Hosseingholizadeh } \\
\text { [18] }\end{array}$ & $\begin{array}{l}\text { Hotspot prediction using } \\
\text { NN }\end{array}$ & Prediction Error & $\begin{array}{l}\text { Pros: Optimize task allocation } \\
\text { Cons: Outcomes not benchmarked }\end{array}$ \\
\hline & $\begin{array}{l}\text { Abbasi \& Kamal } \\
{[19]}\end{array}$ & Neural Network & -Not Discussed & $\begin{array}{l}\text { Pros: Ensure both security and energy } \\
\text { efficiency } \\
\text { Cons: No discussion on outcomes }\end{array}$ \\
\hline & Bahanfar et al. [20] & Neural Network & Energy Consumption & $\begin{array}{l}\text { Pros: Increases lifetime } \\
\text { Cons: Outcomes not benchmarked }\end{array}$ \\
\hline \multirow{6}{*}{ 岂 } & Zarafshan et al. [21 & $\begin{array}{l}\text { Fuzzy Logic using directed } \\
\text { diffusion routing protocol }\end{array}$ & Energy, packets transmitted & $\begin{array}{l}\text { Pros: higher residual energy } \\
\text { Cons: Outcomes not benchmarked }\end{array}$ \\
\hline & Jiang et al. [22] & $\begin{array}{l}\text { Fuzzy-logic-based energy } \\
\text { optimized routing algorithm }\end{array}$ & $\begin{array}{l}\text { Average Residual Energy, } \\
\text { Average energy consumed }\end{array}$ & $\begin{array}{l}\text { Pros: Energy efficient routing decision } \\
\text { Cons: QoS outcomes not discussed }\end{array}$ \\
\hline & Hadjila et al. [23] & $\begin{array}{l}\text { Fuzzy C-means using } \\
\text { centrality and proximity }\end{array}$ & $\begin{array}{l}\text { Alive Nodes, energy } \\
\text { consumption, residual energy }\end{array}$ & $\begin{array}{l}\text { Pros: Outperform LEACH } \\
\text { Cons: Scalability issues not discussed }\end{array}$ \\
\hline & Mostafa et al. [24] & $\begin{array}{l}\text { Stable election protocol } \\
\text { using Fuzzy Logic }\end{array}$ & Energy consumption & $\begin{array}{l}\text { Pros: Outperforms LEACH. } \\
\text { Cons: Impact of proposed technique } \\
\text { not evaluated w.r.t QoS parameters } \\
\text { extensively. }\end{array}$ \\
\hline & Sonmez et al. [25] & $\begin{array}{l}\text { Congestion control using } \\
\text { fuzzy logic }\end{array}$ & $\begin{array}{l}\text { PSNR, frame rate, energy } \\
\text { consumed, average frame loss }\end{array}$ & $\begin{array}{l}\text { Pros: Energy efficient and congestion } \\
\text { free routing } \\
\text { Cons: Not benchmarked with standard } \\
\text { routing protocols for energy efficiency }\end{array}$ \\
\hline & $\begin{array}{l}\text { Behzadi and Azad } \\
\text { [26] }\end{array}$ & $\begin{array}{l}\text { Prioritizing neighbor nodes } \\
\text { to achieve fault tolerant } \\
\text { using Fuzzy Logic }\end{array}$ & Time, mobility & $\begin{array}{l}\text { Pros: effective fault tolerant } \\
\text { Cons: Not benchmarked with standard } \\
\text { routing protocols for energy }\end{array}$ \\
\hline
\end{tabular}




\begin{tabular}{|c|c|c|c|c|}
\hline & & & & efficiency, \\
\hline \multirow{6}{*}{ 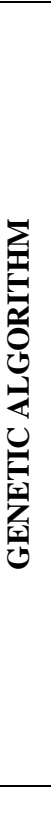 } & Fard et al. [27] & $\begin{array}{l}\text { Multi-Objective Genetic } \\
\text { Algorithm }\end{array}$ & $\begin{array}{l}\text { Packet Arrival rate, energy } \\
\text { consumption, error rate in data } \\
\text { transmission }\end{array}$ & $\begin{array}{l}\text { Pros: better performance compared to } \\
\text { SAR and SPEED } \\
\text { Cons: QoS outcomes not discussed }\end{array}$ \\
\hline & $\begin{array}{l}\text { Liu \& Ravishankar } \\
\text { [28] }\end{array}$ & $\begin{array}{l}\text { Clustering based on genetic } \\
\text { Algorithm }\end{array}$ & Energy & $\begin{array}{l}\text { Pros: Better than LEACH } \\
\text { Cons: QoS outcomes not discussed }\end{array}$ \\
\hline & $\begin{array}{ll}\text { Heidari } & \& \\
\text { Movaghar [29] } & \end{array}$ & $\begin{array}{l}\text { Optimization based on } \\
\text { genetic algorithm }\end{array}$ & Energy, distance & $\begin{array}{l}\text { Pros: Better than LEACH } \\
\text { Cons: QoS outcomes not discussed }\end{array}$ \\
\hline & Zahhad et al. [30] & $\begin{array}{l}\text { Clustering based on genetic } \\
\text { Algorithm }\end{array}$ & Energy & $\begin{array}{l}\text { Pros: Better than LEACH } \\
\text { Cons: QoS outcomes not discussed }\end{array}$ \\
\hline & Mehrjoo et al. [31] & $\begin{array}{l}\text { Combination of genetic } \\
\text { algorithm and bee colony } \\
\text { optimization }\end{array}$ & $\mathrm{CH}$, lifetime & $\begin{array}{l}\text { Pros: Better than LEACH } \\
\text { Cons: QoS outcomes not discussed }\end{array}$ \\
\hline & Authors & Techniques & Performance Parameters & Inference \\
\hline \multirow{7}{*}{ 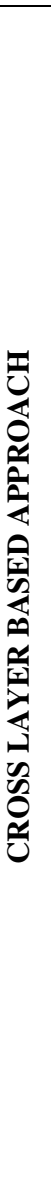 } & Haapola et al. [32] & Cross-layer approach & Energy & $\begin{array}{l}\text { Pros: support energy efficiency in } \\
\text { multi-hop network } \\
\text { Cons: Outcomes not benchmarked }\end{array}$ \\
\hline & Suh et al. [33] & $\begin{array}{l}\text { Interactions between MAC } \\
\text { and Routing layers }\end{array}$ & Energy, & $\begin{array}{l}\text { Pros: Experimented on real-time Mica } \\
\text { Mote, energy efficient } \\
\text { Cons: Unlikely to support large } \\
\text { network }\end{array}$ \\
\hline & Hurni et al. [34] & Cross-layer approach & Delay, energy, & $\begin{array}{l}\text { Pros: support energy efficiency in } \\
\text { multi-hop network } \\
\text { Cons: Outcomes not benchmarked }\end{array}$ \\
\hline & Tsai et al. [35] & $\begin{array}{l}\text { Cross-layer and quorum } \\
\text { based approach }\end{array}$ & Residual energy & $\begin{array}{l}\text { Pros: Better than shortest path routing } \\
\text { protocol } \\
\text { Cons: Should have also benchmarked } \\
\text { with LEACH, QoS outcomes not } \\
\text { discussed }\end{array}$ \\
\hline & Almiani et al. [36] & Energy-aware cross-layer & Energy, & $\begin{array}{l}\text { Pros: Better than Conventional cross- } \\
\text { layer scheduling techniques } \\
\text { Cons: QoS outcomes not discussed }\end{array}$ \\
\hline & $\begin{array}{l}\text { Hamid \& Bashir } \\
\text { [37] }\end{array}$ & Cross-layer approach & $\begin{array}{l}\text { Energy, delay, throughout, data } \\
\text { rate }\end{array}$ & $\begin{array}{l}\text { Pros: Better than AODV } \\
\text { Cons: Should have also compared } \\
\text { with energy efficient routing protocols }\end{array}$ \\
\hline & Espes et al. [38] & Cross-layer approach & Delay, energy, packet loss rate & $\begin{array}{l}\text { Pros: Reduced energy consumption } \\
\text { Cons: Algorithm efficiency with } \\
\text { respect to time and space complexity } \\
\text { not discussed }\end{array}$ \\
\hline \multirow{2}{*}{ 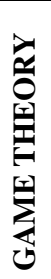 } & Truong et al. [39] & Cooperative game theory & $\begin{array}{l}\text { Lifetime improvement, } \\
\text { deviation }\end{array}$ & $\begin{array}{l}\text { Pros: Provide better load balancing } \\
\text { and network lifetime } \\
\text { Cons: Outcomes not benchmarked }\end{array}$ \\
\hline & $\begin{array}{ll}\text { Koltidas } & \text { and } \\
\text { Pavlidou [40] } & \end{array}$ & $\begin{array}{l}\text { Energy efficient Clustering } \\
\text { using game theory }\end{array}$ & Network lifetime, alive nodes & $\begin{array}{l}\text { Pros: Effective than LEACH } \\
\text { Cons: Outcomes not benchmarked } \\
\text { w.r.t. QoS parameters }\end{array}$ \\
\hline
\end{tabular}




\begin{tabular}{|c|c|c|c|c|}
\hline & $\mathrm{Xu}$ et al. [41] & $\begin{array}{l}\mathrm{CH} \text { selection using game } \\
\text { theory }\end{array}$ & Throughput & $\begin{array}{l}\text { Pros: cost effective clustering } \\
\text { Cons: Less extensive simulation study, } \\
\text { Outcomes not benchmarked }\end{array}$ \\
\hline & Asadi et al. [42] & Voltage & $\begin{array}{l}\text { Average network throughput } \\
\text { for malicious nodes, } \\
\text { Average network utility for } \\
\text { Broadcast, } \\
\text { Average network voltage loss } \\
\text { for malicious nodes. Broadcast }\end{array}$ & $\begin{array}{l}\text { Pros: Minimizes voltage consumption } \\
\text { throughout the network. } \\
\text { Cons: Outcomes not benchmarked }\end{array}$ \\
\hline & Yang and Guan [43] & $\begin{array}{l}\text { Non-cooperative Game } \\
\text { theory }\end{array}$ & $\begin{array}{l}\text { Transmission Success rate, } \\
\text { Energy efficiency, }\end{array}$ & $\begin{array}{l}\text { Pros: Minimizes transmission power } \\
\text { for node, ensure quality of link with } \\
\text { higher success rate } \\
\text { Cons: Not applicable on large network }\end{array}$ \\
\hline & $\begin{array}{l}\text { Rahman and Matin } \\
\text { [44] }\end{array}$ & Particle swarm optimization & Sink location, energy & $\begin{array}{l}\text { Pros: Enhanced lifetime of network } \\
\text { Cons: Outcomes not benchmarked }\end{array}$ \\
\hline 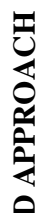 & Loscri et al. [45] & $\begin{array}{l}\text { Particle Swarm } \\
\text { Optimization }\end{array}$ & Mean energy, node position & $\begin{array}{l}\text { Pros: Better coverage and less energy } \\
\text { consumption } \\
\text { Cons: Algorithm complexity not } \\
\text { discussed, no benchmarking with } \\
\text { standard energy efficient protocols }\end{array}$ \\
\hline 空 & $\begin{array}{l}\text { Poostfroushan et al. } \\
\text { [46] }\end{array}$ & $\begin{array}{l}\text { energy efficient backbone } \\
\text { formation algorithm using } \\
\text { particle Swarm } \\
\text { Optimization }\end{array}$ & Energy & $\begin{array}{l}\text { Pros: Effective energy preservation } \\
\text { Cons: No benchmarking outcomes }\end{array}$ \\
\hline 疍 & $\begin{array}{l}\text { Zhong and Zhang } \\
\text { [47] }\end{array}$ & Ant Colony Optimization & $\begin{array}{l}\text { Mean network lifetime, mean } \\
\text { running time }\end{array}$ & $\begin{array}{l}\text { Pros: Enhance lifetime } \\
\text { Cons: No discussion on spatial } \\
\text { relationship of the mobile sink, doesn't } \\
\text { support multiple mobile sink }\end{array}$ \\
\hline 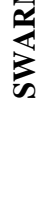 & Ping \& Yan [48] & Ant Colony Optimization & Residual Energy & $\begin{array}{l}\text { Pros: Enhance Lifetime } \\
\text { Cons: Less extent of simulation study, } \\
\text { no discussion of algorithm complexity, } \\
\text { no benchmarking with energy efficient } \\
\text { protocols }\end{array}$ \\
\hline
\end{tabular}

\subsection{Cross Layer-based Approach}

Literatures have also witness various studies using cross-layer approach, where the emphasis was given to physical layer, link layer, and network layer. Haapola et al. [32], Suh et al. [33], and Hurni et al. [34] have performed energy efficiency using MAC, radio transceiver energy, and cross layer approach to maintain optimal lifetime. Tsai et al. [35] have proposed a framework for energy conservation on MAC layer for finding the query paths based on energy cost consumed in grid quorums. Cross layer approach was also used for energy efficiency in data aggregation process in wireless sensor network as seen in the work of Almiani et al. [36]. The study has integrated clustering protocols, MAC, and routing to minimize overhead caused due to transmission schedules. Hamid and Bashir [37] have a cross-layer approach for real-time data delivery to provide an efficient interaction between delay, admission control, dynamic duty cycle, and routing. Most recently, Espes et al. [38] have adopted cross-layer approach to energy efficiency along with low delay and high data rate.

\subsection{Game Theory}

Owing to the potential implication of strategic decision making principles, game theory was found to be adopted more frequently in last 5 years. Truong et al. [39] have adopted cooperative game theory to solve the energy issues of coverage factor in wireless sensor network. Another significant study for Koltidas [40], Xu et al. [41], Asadi et al. [42] were seen to use game theory to perform energy efficient clustering. Yang and Guan [43] have also adopted non-cooperative game theory for accomplishing energy efficiency in wireless sensor network.

\subsection{Swarm Intelligence}

Apart from the above discussed techniques, swarm intelligence techniques were also adopted for accomplishing energy efficiency in wireless sensor network. The potential feature of best solution exploration process mimicked from the biological objects are quite innovative mathematical idea, which was found to adopted by the research community from last decade in wireless sensor network. Some of the frequently used swarm 
intelligent techniques include ant colony optimization, bee colony optimization, particle swarm optimization etc. Rahman and Matin [44], Loscri et al. [45], and Poostfroushan et al. [46] have used particle swarm optimization for locating the best position of sink for achieving energy efficiency. Zhong [47] and Ping [48] have used ant colony optimization for enhancing the network lifetime using mobile sink.

\section{RESEARCH GAP}

The present paper has reviewed only the significant research contribution done most recently. This paper has not considered the any manuscript available online that has repeated discussion on same techniques or algorithms to avoid redundancy in content development of this paper. Hence, out of massive archival of implementation studies contributed by various researchers, it can be seen that every techniques have their own advantages as well as limitations. This section will briefly discuss about the potential research gap identified after reviewing the prior literatures illustrated in this manuscripts:

\subsection{Few Benchmarked Studies}

It was found that majority of the existing studies have discussed their outcomes without any aid of benchmarked studies done till date. Table 2 as well as Table 3 highlights list of studies where majority of the techniques were discussed and published without any benchmarking. Hence, it becomes quite a difficult task for the upcoming researcher to understand the effectiveness of the discussed technique. Moreover, certain studies e.g. Mao et al. [4], Francesco et al. [7], Tang et al. [8], Sonmez et al. [25], Behzadi and Azad [26],Tsai et al. [35], Hamid \& Bashir [37] etc have adopted specific benchmarking of their techniques. It is suggested that if the researcher is adopting a techniques to improve energy efficiencies, it is essential that they must compared their outcomes with the most standard energy efficient routing (e.g. LEACH, PEGASIS etc) protocols rather than arbitrary selecting other specific techniques for benchmarking.

\subsection{Less study on Algorithm Efficiency}

Majority of the studies have chosen to showcase the performance parameters like packet delivery ratio, energy, delay, throughput, etc to highlight the performance of their techniques. However, question arises whether the algorithm is applicable when the scenario changes. Studies performed by Guan \& Li [17], Hosseingholizadeh [18], Abbasi \& Kamal [19], Bahanfar et al. [20] have potential issues of proving the algorithm efficiency as the technique they adopted have time consuming training process, which cannot be applied on time and mission critical applications of wireless sensor network.

\subsection{Scalability}

Majority of the studies discussed in Table 2 and Table 3 are more inclined towards small scale wireless sensor network. However, real-time applications of wireless sensor network calls for deployment of sensor nodes in large geographic region using maximum number of sensor nodes, whereas majority of the studies are tested on 50-200 sensor nodes. Hence, the scalability issues of wireless sensor network are actually not met by the existing techniques of energy conservation.

\subsection{Less Adaptability}

Adaptability of the technique is one of the most important criteria of any applications of wireless sensor network. There are various cases of applications e.g. forest fire detection, moving object detection, etc, where it is required to understand and interpret the data being captured in the dynamically changing environment without any apriori information. The studies performed by Zarafshan et al. [21], Jiang et al. [22], Hadjila et al. [23], Mostafa et al. [24], Sonmez et al. [25], Behzadi and Azad [26], although proposes some of the unique techniques of preserving energy, but couldn't address the issues of adaptability and dynamic environment. The techniques are less prone to adapt to changing environment as they are based on rule design. The studies performed by Fard et al. [27], Liu \& Ravishankar [28], Heidari \& Movaghar [29], Zahhad et al. [30], and Mehrjoo et al. [31] have adopted evolutionary algorithm that can essentially scale well to higher dimensional problems of energy efficiencies, but majority of the techniques adopted using evolutionary approach considers clustering techniques only. Hence dynamic adaptability charecteristics that are highly required in the cases of mobility in wireless sensor network are eventually not met is the existing system.

\subsection{Sophisticated and Complex Techniques}

Various studies done in the past have adopted complex design process where the design process doesn't actually meet the realtime requirements of the applications. The studies performed by Truong et al. [39], Koltidas and Pavlidou [40], Xu et al. [41], Asadi et al. [42], Yang and Guan [43] have adopted complex strategic decision making principles using game theory by considering less practical assumptions of the sensor node's knowledge. However, in the process of data aggregation as well as data fusion there are various complicated problems like identifying the redundancies, data packet collision, nonconventional selection criteria of $\mathrm{CH}$, resisting the phenomenon of re-transmission, intra and inter clustering mechanism, which were quite difficult to be addressed using game theory.

\subsection{Impact on QoS Parameters}

Quality of Service parameter plays a critical role in understanding the effect of discussed techniques for energy preservation. It can be seen that some of the studies e.g. Fard et al. [27], Heidari \& Movaghar [29] , Zahhad et al. [30], Mehrjoo et al. [31], Tsai et al. [35], Almiani et al. [36], Koltidas and Pavlidou [40] etc to name a few have considered only energy parameters and ignored the possible impact of their techniques on bandwidth, jitter, interarrival time, latency, packet delivery ratio, throughput, etc. If the QoS parameters are not analyzed, it is quite possible that the application could not meet the service requirements for which it is meant for. Hence, a trade-off in theoretical (or analytical) approach with real-time requirement is identified in the study of energy preservation techniques till date.

\section{CONCLUSION}

There are already massive survey papers that have been published till date speaking of various techniques of energy conservation techniques of wireless sensor network. It will be found that all the survey papers published till date have discussed the techniques theoretically, which has one advantage as one disadvantage too. The advantage of existing survey papers published till date are give more theoretical description of the techniques, which will enable the reader to understand the techniques very vividly. The disadvantage of the existing survey paper published till date are that it have never discussed about the in-depth analysis of effectiveness of prior research attempt, for which reason the readers will not be able to compare and find out which is the most suitable technique that they should adopt in their study. Hence, it is quite necessary to talk about both advantages as well as limitation of the prior studies more discrete to make the research community to understand the need of more effective techniques. The proposed paper has presented a state-of-art review of literature that has discussed the most recent research attempts and closely studied the potential advantages as well as constraints of their outcomes. We can state that energy efficiency in wireless sensor network has no doubt have potential algorithms, but 
applicability of such frequently adopted algorithms in various scenarios are still in infancy stage. The research gap identified in this paper has elicited more facts about the findings of the prior research attempt. Our study will be continued in further direction to mitigate the research gap identified in this paper and thereby solving the energy conservation issues in wireless sensor network. Scalability, benchmarking, and algorithm efficiency are the core motives of our future work.

\section{REFERENCES}

[1] R. Faludi, R.2010. Building Wireless Sensor Networks: with ZigBee, XBee, Arduino, and Processing.O'Reilly Media, Inc.", Computers., 322 pages, 2010

[2] Heinzelman, W. R., Chandrakasan, A., and Balakrishnan, H.2000. Energy-Efficient Communication Protocol for Wireless Microsensor Networks. IEEE Proceedings of the Hawaii International Conference on System Sciences

[3] Gupta, A., Nayyar, A.2014. A Comprehensive Review of Cluster-Based Energy Efficient Routing Protocols in Wireless Sensor Networks. International Journal of Research in Computer and Communication Technology. Vol. 3, Issue. 1

[4] Mao, X., Tang, S., Xu, X., Li, X-Y., Ma, H.2011. Energy Efficient Opportunistic Routing in Wireless Sensor Networks. IEEE Transactions On Parallel And Distributed Systems. vol.22, Iss.11, pp.1934-1942

[5] Biswas, S., and Morris, R.2005. ExOR: Opportunistic MultiHop Routing for Wireless Networks. ACMSIGGCOM-Proceedings of conference on Application, technologies, architectures, and protocols for computer communication, Vol. 35, Iss.4, pp.133-144

[6] Halkes, G.P., and Langendoen, K.G.2007. Crankshaft: An Energy-Efficient MAC-Protocol for Dense Wireless Sensor Networks. Springer-Verlag. pp. 228-244

[7] Francesco, M. D., Shah, K., Kumar, M., and Anastasi, G. 2010. An Adaptive Strategy for Energy-Efficient Data Collection in Sparse Wireless Sensor Networks. SpringerVerlag, pp. 322-337

[8] Tang, L., Sun, Y., Gurewitz, O., Johnson, D. B.2011. EMMAC: A Dynamic Multichannel Energy-Efficient MAC Protocol for Wireless Sensor Networks, ACM-MobiHoc

[9] Aziz, S.M., Pham, D.M.2013. Energy Efficient Image Transmission in Wireless Multimedia Sensor Networks. IEEE communications letters. Vol. 17, No. 6

[10] Long, J., Dong, M., Ota, K., and Liu, A.2014. Achieving Source Location Privacy and Network Lifetime Maximization Through Tree-Based Diversionary Routing in Wireless Sensor Networks. IEEE Access, Vol.2

[11] Islam, A. B. M. A. A., Hossain, M. S., Raghunathan, V., and Charlie, H.2014.Backpacking: Energy-Efficient Deployment of Heterogeneous Radios in Multi-radio HighData-Rate Wireless Sensor Networks. IEEE Access, Iss.99

[12] Takaishi, D., Nishiyama, H., Kato, N., and Miura, R.2014. Towards Energy Efficient Big Data Gathering in Densely Distributed Sensor Networks.IEEE Transactions on Emerging Topics in Computing, Iss.99

[13] Damaso, A., Freitas, D., Rosa, N., Silva, B., and Maciel, P.2013. Evaluating the Power Consumption of Wireless Sensor Network Applications Using Models. Sensors, Vol. 13, pp.3473-3500

\section{[14] http://cpntools.org/}

[15] Ashwini, K. B., and Raju, G. T.2014. Extending Network Lifetime by Time-Constrained Data Aggregation in Wireless Sensor Networks. Advances in Intelligent Systems and Computing, DOI: 10.1007/978-81-322-1665-0_2

[16] Manzoor, B., Javaid, N., Rehman, O., Akbar, M., Nadeem, Q., Iqbal, A., Ishfaq, M.2013. Q-LEACH: A New Routing Protocol for WSNs. Elsevier

[17] Guan, P., and Li, X.2007. Minimizing Distribution Cost of Distributed Neural Networks in Wireless Sensor Networks. $50^{\text {th }}$ Annual IEEE Global Telecommunications Conference (GLOBECOM), Washington DC

[18] Hosseingholizadeh, A.2009. A Neural Network approach for Wireless sensor network power management. Proc. $28^{\text {th }}$ IEEE Inter. Symp. on Reliable Distributed Systems, USA

[19] Abbasi, A. A., Kamal, A.2011. An Intelligent NeuralWireless Sensor Network Based Schema for Energy Resources Forecast. International Journal of Advanced Science and Technology, Vol. 33

[20] Bahanfar, S., Kousha, H., and Darougaran, L.2011. Neural networks for error detection and data aggregation in wireless sensor network. International Journal of Computer Science Issues, Vol. 8, Issue 5, No 3

[21] Zarafshan, F., Karimi, A., Al-Haddad, S. A. R.2009. A Novel Fuzzy Diffusion Approach for Improving Energy Efficiency in Wireless Sensor Networks. ICEE

[22] Jiang, H., Sun, Y., Sun, R., and Xu, H.2013. Fuzzy-LogicBased Energy Optimized Routing for Wireless Sensor Networks. Hindawi Publishing Corporation International Journal of Distributed Sensor Networks, Article ID 216561, pp. 8

[23] Hadjila, M., Guyennet, H., Feham, M.2013. A Routing Algorithm based on Fuzzy Logic Approach to Prolong the Lifetime of Wireless Sensor Networks. International Journal of Open Scientific Research, Vol.1, No. 5, 24-35

[24] Mostafa, B., Saad, C., and Abderrahmane, H.2014. Fuzzy Logic Approach to Improving Stable Election Protocol for Clustered Heterogeneous Wireless Sensor Networks. International Journal of Computer Science and Network Security, Vol.14, No.1

[25] Sonmez, C., Incel, O. D., Isik, S., Donmez, M. Y., and Ersoy, C. 2014. Fuzzy-based congestion control for wireless multimedia sensor networks. EURASIP Journal on Wireless Communications and Networking, Vol.63

[26] Behzadi, S., Azad, M.2014. Fault-tolerant in wireless sensor networks using fuzzy logic. International Research Journal of Applied and Basic Sciences, Vol. 8, Iss.9, pp.1276-1282

[27] Fard, G. H. E., Monsefi, R., Akbarzadeh, M-R., Yaghmaee, M. H.2010. A Multi-objective Genetic Algorithm based Approach for Energy Efficient QoS-Routing in Two-tiered Wireless Sensor Networks. $5^{\text {th }}$ IEEE International Symposium on Wireless Pervasive Computing, pp.80-85

[28] Liu, J-L., and Ravishankar, C. V.2010. LEACH-GA: Genetic Algorithm-Based Energy-Efficient Adaptive Clustering Protocol for Wireless Sensor Networks. International Journal of Machine Learning and Computing, Vol.1, Iss.1, pp.79-85 
[29] Heidari, E., and Movaghar, A.2011. An efficient method based on genetic algorithms to solve sensor network optimization problem. International journal on applications of graph theory in wireless ad hoc networks and sensor networks. Vol.3, No.1

[30] Zahhad, M. A., Ahmed, S. M., Sabor, N., and Sasaki, S.2014. A New Energy-Efficient Adaptive Clustering Protocol Based on Genetic Algorithm for Improving the Lifetime and the Stable Period of Wireless Sensor Networks. International Journal of Energy, Information and Communications. Vol.5, Issue 3, pp.47-72

[31] Mehrjoo, S., Aghaee, H., Karimi, H.2011. A Novel Hybrid GA-ABC based Energy Efficient Clustering in Wireless Sensor Network. Canadian Journal on Multimedia and Wireless Networks. Vol. 2, No. 2

[32] Haapola, J., Shelby, Z., Raez, C. P., and Mahonen, P.2005. Cross-layer Energy Analysis of Multi-hop Wireless Sensor Networks. Proceedings of EWSN, pp.33-44

[33] Suh, C., Ko, Y-B., and Son, D-M.2006. An Energy Efficient Cross-Layer MAC Protocol for Wireless Sensor Networks. Springer-Verlag, pp. 410-419

[34] Hurni, P., Braun, T., Bhargava, B. K., Zhang, Y.2008. Multi-Hop Cross-Layer Design in Wireless Sensor Networks: A Case Study. IEEE International Conference on networking and communication

[35] Tsai, C-H., Hsu, T-W., Pan, M-S., and Tseng, Y-C.2009. Cross-Layer, Energy-efficient Design for Supporting Continuous Queries in Wireless Sensor Networks: A Quorum-Based Approach.Wireless personal communications, Vol.51, Iss.3, pp.411-426

[36] Almiani, K., Selvakennedy, S., and Viglas, A.2008. RMC: An Energy-Aware Cross-Layer Data-Gathering Protocol for Wireless Sensor Networks. 22nd IEEE International Conference on Advanced Information Networking and Applications, pp.410-417

[37] Hamid, Z., and Bashir, F.2013. XL-WMSN: cross-layer quality of service protocol for wireless multimedia sensor networks. EURASIP Journal onWireless Communications and Networking

[38] Espes, D., Lagrange, X., Suarez, L.2014. A cross-layer MAC and routing protocol based on slotted Aloha for
Wireless Sensor Networks. Springer annals of telecommunications. pp.ISSN 0003-4347

[39] Truong, C. D., Khan, M. A., Sivrikaya, F.2010. Cooperative Game Theoretic Approach to Energy-Efficient Coverage in Wireless Sensor Networks. IEEE International Conference on Networked Sensing System, pp.73-76

[40] Koltsidas, G., and Pavlidou, F-N.2011. A Game Theoretical Approach to Clustering of Ad-Hoc and Sensor Networks", Telecommunication Systems, Vol. 47, No 1-2, pp. 81-93, DOI: $10.1007 / \mathrm{s} 11235-010-9303-5$

[41] Xu, Z., Yin, Y., Chen, X., and Wang, J.2013. A Gametheory Based Clustering Approach for Wireless Sensor Networks. NGCIT-ASTL, Vol. 27, pp. 58 - 66

[42] Asadi, M., Zimmerman, C., and Agah, A.2013. A Gametheoretic Approach to Security and Power Conservation in Wireless Sensor Networks. International Journal of Network Security. Vol.15, No.1, pp.50-58

[43] Yang, G., and Guan, X.2014. A Non-cooperative Game Theoretic Approach to Energy-efficient Power Control in Wireless Sensor Networks. International Journal of Future Generation Communication and Networking. Vol.7, No.1, pp.169-180

[44] Rahman, M.N., Matin, M.A.2011. Efficient Algorithm for Prolonging Network Lifetime of Wireless Sensor Networks. IEEE- Tsinghua Science And Technology, Vol.16, No.6, pp.561-568

[45] Loscrí, V., Natalizio, E., Guerriero, F., Aloi, G.2012. Particle Swarm Optimization Schemes Based on Consensus for Wireless Sensor Networks. ACM

[46] Poostfroushan, S., Sarram, M. A., and Sheikhpour, R.2014. Energy Efficient Backbone Formation Using Particle Swarm Optimization Algorithm in Wireless Sensor Networks", International Journal of Grid and Distributed Computing, Vol.7, No.1, pp.123-134

[47] Zhong, J-H., and Zhang, J.2012. Ant Colony Optimization Algorithm for Lifetime Maximization in Wireless Sensor Network with Mobile Sink. ACM

[48] Ping, W.J., Yan, L. J.2013. Wireless Sensor Network Mobile Agent routing based on the Improved Ant Colony Algorithm. Journal of Convergence Information Technology. Vol.8, No. 5 